\title{
MedienPädagogik
}

www. medienpaed.com

Zeitschrift für

Theorie und Praxis

der Medienbildung

ISSN 1424-3636

Themenheft Nr. 18: Neue Medien und individuelle Leistungsdarstellung Möglichkeiten und Grenzen von ePortfolios und eAssessments

\section{Implikationen aus dem gegenwärtigen Mediennutzungsverhalten Lernender}

\author{
Chancen und Grenzen von ePortfolios zur persönlichen Markenbildung
}

Michael J. Eble ${ }^{1}$

\begin{abstract}
Bei der Betrachtung des Mediennutzungsverhaltens Jugendlicher und junger Erwachsener in Deutschland zeigt sich eine zunehmend stärkere Integration von Online-Angeboten in den Alltag. Ein zentrales Element dieses Nutzungsverhaltens ist die Online-Kommunikation mittels Social Networks. Verschiedene und voneinander unabhängige Studien zeichnen das Bild einer häufigen und intensiven Nutzung dieser Angebote. Dabei stellen sich die Mitglieder dieser Online-Gemeinschaften innerhalb der Plattformen über individuelle Profile dar. Im Fokus steht für die Nutzer hier die authentische Selbstdarstellung. Somit können Profile als zumindest teilweise Darstellung der eigenen Identität angesehen werden. Die Definition derselben ist gleichzeitig Grundlage des identitätsbasierten Ansatzes der Markenbildung. Dazu zählt die Festlegung der eigenen Herkunft, Kompetenzen, Werte, Persönlichkeit, Leistungen und Ziele. Hier wird - wie auch in Social Networks üblich - ein regelmässiger Abgleich von Selbst- und Fremdbild durchgeführt.

Die Kombination von ePortfolios (im Stil der Profilierung auf Social Networks) mit dem identitätsbasierten Ansatz der Markenbildung kann in formellen Lehr- und Lernkontexten nutzbar gemacht werden. Auf Basis habitualisierter Selbstdarstellung und Reflexion kann die eigene Lern- und Leistungsentwicklung abgebildet und kontinuierlich beobachtet werden. Somit können ePortfolios Lernenden ein Werkzeug sein, um selbstgesteuert die eigene Ziel- und Ist-Positionierung zu bestimmen und zu analysieren. Sie können damit als Instrument die persönliche Markenbildung in diesem Segment unterstützen.
\end{abstract}

Der Autor dankt den anonymen Gutachtern für wertvolle Hinweise und Kommentare zu einer früheren Fassung dieses Aufsatzes. 


\section{Einführung}

Das World Wide Web ist von einem dynamischen Entwicklungsprozess gekennzeichnet: Zunehmend mehr Menschen in Deutschland sind immer längere Zeit im Internet ${ }^{2}$ unterwegs. Seine gesellschaftliche Bedeutung wächst. Zudem ist ein Verschwimmen der klaren Trennung von Produzenten ${ }^{3}$ und Rezipienten zu beobachten. Ein zentrales Element dieser Entwicklung sind Communitys, die ein starkes Mitgliederwachstum verzeichnen. Auch für Jugendliche und junge Erwachsene ist 〈das Internet〉 zu einem wichtigen Bestandteil ihres Alltags geworden (vgl. van Eimeren/Frees 2008). Zudem zeigt sich, dass die Heranwachsenden neue Dienste häufig überdurchschnittlich schnell adaptieren (vgl. Fisch/Gscheidle 2008b). Gegenwärtig steht so besonders das Thema ‘Vernetzungs im Fokus der Onliner: Dabei handelt es sich einerseits um die Vernetzung von Menschen untereinander und andererseits um die Vernetzung von Inhalten. In Social Networks, einer Form von Communitys (vgl. Schmidt/Paus-Hasebrink/Hasebrink/Lampert 2009, 9f.), erstellen Nutzer persönliche Profilseiten und hinterlegen Informationen über ihre Hobbys und Interessen.

Grundsätzlich können, von dieser Exposition ausgehend, derartige Plattformen für selbstbestimmte Lern- und Leistungsdarstellungen ergänzt und als ePortfolios genutzt werden. Dabei wird der Begriff «ePortfolios in diesem Beitrag in Anlehnung an Häcker (2005) wie folgt verstanden: Ein ePortfolio besteht aus digitalen Informationen, die die Kompetenzen und Leistungen eines Lernenden darstellen. Es dient damit dem Zweck - neben dem jeweils gegenwärtigen Ist-Zustand - die Entwicklung in einzelnen Bereichen systematisch abzubilden und daraus entsprechende Handlungsschritte abzuleiten. Somit ist es «ein Instrument zur Kompetenzdarstellung» (Häcker 2005, 5) und zur zielorientierten Planung.

Häcker (ebd.) sieht als Basis der Leistungsdarstellung mittels Portfolios drei Prinzipien:

- Kommunikation

- Partizipation

- Transparenz

So könnten Portfolios «gleichzeitig als Lehr-Lern-Instrument und [Hervorhebung im Original] als (Selbst-)Beurteilungsinstrument eingesetzt werden» (Häcker 2005, 4) und rückten eine Kompetenzorientierung statt einer Defizitorientierung in den Vordergrund (siehe ebd.).

2 Im Folgenden werden die Begriffe 〈Internet〉 und 〈Web〉 synonym verwendet, die technische Abgrenzung wird vernachlässigt.

3 Im vorliegenden Beitrag wird ausschliesslich die männliche Form bei Artikeln und Endungen verwendet. Diese soll stellvertretend für beide Geschlechter verstanden werden. 
Aus diesen Überlegung lassen sich drei Fragen ableiten: Wie kann diese Auseinandersetzung mit der eigenen Person und Identität als ein Schritt zu einer persönlichen Markenbildung genutzt werden? Welcher Bezug besteht zwischen persönlicher Markenbildung und formellen Lehr- bzw. Lernkontexten? Wie können Adoption und Akzeptanz von ePortfolios unterstützt werden?

Der kommunikationswissenschaftliche Beitrag untersucht anhand dieser Fragen Chancen und Grenzen von ePortfolios. Dabei geht er vom Mediennutzungsverhalten Lernender aus und rekurriert auf den identitätsbasierten Ansatz der Markenbildung. Der Beitrag basiert auf Sekundärforschung und berücksichtigt Erkenntnisse aus Medientheorie sowie Marketing-, Markt- und Sozialforschung.

\section{Nutzung von Social Networks}

Bei der Betrachtung von Social Networks fällt auf, das diese ein zentrales Element des «neuens Internets sind. Dessen Bedeutungswandel wird häufig mit dem Begriff «Web 2.0 beschrieben. Wie Schmidt et al. $(2009,5)$ ausführen, ziehe das Web (2.0) seinen «Wert vor allem daraus, dass man mit anderen Menschen kommuniziert und interagiert». Dementsprechend und vor dem Hintergrund, dass keine neue Version (2.0 statt 1.0) des Internets (erschienen) und gemeint sei, so Schmidt et al., sei der Begriff «Social Web〉 dem des «Web 2.0) vorzuziehen (vgl. Schmidt et al. 2009, 5). Da aus Sicht einer sozialwissenschaftlich orientierten Medien- und Kommunikationswissenschaft vor allem Nutzung durch und Wirkung auf Individuum und Gesellschaft gegenständlich sind (so auch die These hier), wird diesem Begriffsvorschlag im vorliegenden Beitrag gefolgt. Aus medien- und kommunikationswissenschaftlicher Perspektive kann der Terminus daher wie folgt verstanden werden: «Social Web〉 bezeichnet die mittels intuitiv nutzbarer Technik vereinfachte Einschreibung, nachhaltige Abbildung und dynamische Beeinflussung sozialer Strukturen und Prozesse im Internet (siehe Eble 2009).

Diese Aspekte finden sich besonders in Social Networks. Dabei wird die personenbezogene Vernetzung von jüngeren Onlinern besonders intensiv genutzt: Wie van Eimeren/Frees $\left(2008\right.$, 336) in der Auswertung der ARD/ZDF-Onlinestudie $2008^{4} z e i-$ gen, waren 56 Prozent der 14- bis 29-jährigen Surfer im Jahr 2008 mindestens einmal wöchentlich in einer Online-Community aktiv. Dementsprechend haben Social Networks in den vergangenen Jahren einen starken Nutzungszuwachs verzeichnet. In Deutschland existieren z. B. Facebook, StudiVZ und Xing. Die Angebote haben gemeinsam, dass angemeldete Nutzer dort Profile über die eigene Person erstellen und untereinander vernetzen können. Profilseiten sind, netzwerktheoretisch formuliert, Knoten, Verbindungen zwischen ihnen Kanten (vgl. Stegbauer 2008,

\footnotetext{
${ }^{4}$ Grundgesamtheit: Alle bundesdeutschen Erwachsenen ab 14 Jahren. Ausgangsstichprobe: 2.574 Personen. Mit 1.802 Personen konnte ein komplettes Interview durchgeführt werden. 1.186 Personen wurden als «zumindest gelegentliche Internetanwender» erfasst, 616 Interviewte hatten keinen Zugang zum Internet. Die Erhebung basiert auf Selbsteinschätzung und Eigenauskunft. Basis: Onlinenutzer ab 14 Jahre in Deutschland 2008, N=1.186, soweit nicht anders angegeben.
} 
166). Ausserdem stehen den Mitgliedern verschiedene Kommunikationsmöglichkeiten zur Verfügung.

Dabei lassen sich drei Komponenten als Voraussetzung zur Nutzung des Social Webs identifizieren: Dazu gehören die Ausstattung mit Breitbandinternetzugängen, zeit- und volumenunabhängige Tarife (Flatrates) sowie ausreichend leistungsstarke Endgeräte. Mehrere Quellen zeigen, dass diese drei Kriterien in der Bundesrepublik Deutschland grundsätzlich in weitem Mass erfüllt sind (vgl. Fisch/ Gscheidle 2008a; van Eimeren/Frees 2008; Wirtz 2008).

Das gilt nach Ergebnissen der Studienreihe Jugend, Information, (Multi-) Media $(\mathrm{JIM} \text {-Studie })^{5}$ auch für die Gruppe der Jugendlichen und jungen Erwachsenen im Speziellen: Privathaushalte, in denen Jugendliche leben, verfügen fast vollständig über Computer und Internetzugang (vgl. MPFS 2008, 8). Davon machten 62 Prozent der Jugendlichen täglich Gebrauch, weitere 22 Prozent zumindest mehrmals pro Woche. Montags bis freitags verbrächten die jugendlichen Onliner nach eigener Einschätzung durchschnittlich 120 Minuten pro Tag im Internet (ebd., 46f). Als Anwender von Angeboten des Social Webs weist die Studie 84 Prozent der jugendlichen Internetnutzer aus, 62 Prozent sind in diesem Bereich täglich oder zumindest regelmässig ${ }^{6}$ aktiv (MPFS 2008, 50; vgl. auch Fisch/Gscheidle 2008b sowie van Eimeren/Frees 2008).

Vier von zehn der 12- bis 19-jährigen Onliner steuern täglich Social Networks an, weitere 16 Prozent greifen auf z. B. Facebook zumindest mehrmals pro Woche zu (vgl. MPFS 2008, 54; vgl. auch Fisch/Gscheidle 2008b, 362) ${ }^{7}$. Nach Schmidt et al. $(2009,6)$ besuchen 69 Prozent der 12- bis 24-jährigen Onliner ${ }^{8}$ mehrmals pro Woche derartige Angebote. Zu ähnlichen Ergebnissen kommt eine Studie des Bundesverbandes Informationswirtschaft, Telekommunikation und neue Medien (BITKOM): Die repräsentative Befragung spiegelt eine nachhaltige Integration von OnlineKommunikation in den Alltag von Jugendlichen wider (vgl. BITKOM 2009).

Social Networks werden als zentraler Wachstumstreiber der Aktivitäten im Social Web gesehen - zumal im Bereich der «klassischen` Medien kein äquivalentes Angebot zu finden ist (vgl. MPFS 2008, 50; Haas/Trump/Gerhards/Klingler 2007, 217f). Als Motiv hinter der Nutzung lassen sich mit Hug/Hipfl $(2006,17)$ Vertrautheit und emotionale Bindung zu Bezugspersonen vermuten, eine "geteilte Art des Den-

5 Grundgesamtheit: sieben Millionen Jugendliche im Alter von 12 bis 19 Jahren in Telefon-Haushalten der Bundesrepublik Deutschland (vgl. MPFS 2008, 4). Basis: Alle Befragten, N=1.208, soweit nicht anders angegeben.

6 «Regelmässig» sei hier im Sinne der JIM-Studie gleichbedeutend mit «mindestens mehrmals pro Woche» (MPFS 2008, 6).

7 In der JIM-Studie wurde Facebook bisher, vermutlich auf Grund des späten Eintritts in den deutschen Markt, nicht genannt, stattdessen u. a. SchülerVZ. Es kann davon ausgegangen werden, dass in zukünftigen Ausgaben der Studie von den Befragten auch Facebook angegeben wird.

8 Die entsprechende Studie ist eine Repräsentativbefragung unter Onlinenutzerinnen und -nutzern im Alter von 12 bis 24 Jahren in der Bundesrepublik Deutschland. N=650. Vgl. Schmidt et al. 2009, 5 . 
kens» (Hug/Hipfl 2006, 3) schafft Verbindungen zwischen Mitgliedern von Social Networks. Dort thematisierte Ereignisse oder Sachverhalte können auch abseits der Communitys zur Gesprächsgrundlage werden, also Anschlusskommunikation (vgl. Jäckel 2005, 181ff; Kroeber-Riel/Weinberg 2003, 666ff) auslösen (vgl. Eble 2009). Dadurch kann die Teilhabe an Social Networks Bedeutung für die gesellschaftliche Integration erlangen (vgl. Schmidt et al. 2009, 15). Dies kann als Erklärung dafür dienen, warum die aktive Beteiligung in Communitys intensiver ist als in anderen Angeboten des Social Webs (vgl. Fisch/Gscheidle 2008b sowie Feierabend/Kutteroff 2008, 621).

\section{Selbstdarstellung in Social Networks}

Social Networks werden von ihren Mitgliedern auch zur Selbstdarstellung verwendet (vgl. Fisch/Gscheidle 2008b, 363). So beobachten etwa Schmidt et al. (2009), dass diese Plattformen zum Identitäts- und Beziehungsmanagement genutzt werden. Jugendliche sammeln hier regelmässig Erfahrungen mit computervermittelter Kommunikation und bilden Kompetenzen aus, die eigene Person mittels Profilseiten in den einzelnen Netzwerken darzustellen. Dabei bestehen die Formulare zur Erstellung und Pflege der Profile hauptsächlich aus Textfeldern, mittels derer u. a. Geschlecht, Geburtsdatum, Wohnort, Beziehungsstatus, politische Einstellung, religiöse Ansichten, Interessen, Lieblingsmusik, Schule, Hochschule und Beruf sowie Kontaktdaten angegeben werden können. Durch die Vorgaben in den Profilmasken, so beobachten Schmidt et al. $(2009,11)$, sei die Selbstdarstellung standardisiert. Eine solche Form lässt sich in besonderem Masse in Business-Communitys wie Xing beobachten: Hier sind die Funktionen zur Selbstdarstellung stark sachlich ausgerichtet und strukturiert. Zu berücksichtigen ist dabei, dass Xing in den genannten Erhebungen nicht gegenständlich war und als Zielgruppe Jugendliche und junge Erwachsene nur eingeschränkt adressiert (vgl. Xing AG 2009). Im Hinblick auf z. B. StudiVZ stünde die Standardisierung in einem Spannungsverhältnis zu dem Bedürfnis der Onliner ihre «persönliche und spezifische Identität auf den Plattformen auszudrücken und sichtbar zu machen» (Schmidt et al. 2009, 11). Folgt man dieser Sicht, so könnte eine stärkere Modularisierung und Flexibilisierung der Profilseiten den Nutzern entgegenkommen. Andererseits kann sich dadurch die Komplexität der Anwendung erhöhen und die Bedienung entsprechend schwieriger werden.

Jugendlichen geht es bei Profilerstellung und -verknüpfung offenbar «vor allem um Authentizität - die potenziell vorhandene Möglichkeit, eine andere Identität anzunehmen oder das eigene Aussehen auszublenden, stösst nur verhalten auf Zustimmung» (MPFS 2008, 56). Ähnliches beobachten Schmidt et al. (2009, 14): Den befragten 12- bis 24-jährigen Onlinern sei es wichtig, Aspekten der eigenen Person Ausdruck zu verleihen, die Pflege der eigenen Profilseite sei die «wichtigste Form des Identitätsmanagements im Social Web». In einer Untersuchung zu Iden- 
tität und Selbstdarstellung in Chats kommen Scherer/Wirth (2002) zu ähnlichen Ergebnissen (vgl. auch Höflich/Gebhardt 2001).

Für die Erstellung und Pflege ihrer Profilseiten sowie zum Austausch mit anderen Community-Mitgliedern messen 12- bis 19-jährige Onliner Fotos einen hohen Wert zu (MPFS 2008, 55; vgl. auch Schmidt et al. 2009, 14): Gestützt von Bildern laufen Prozesse des Kennenlernens und Annäherns ab. Eindrücke von z. B. Partys werden als Fotografien festgehalten und in virtuellen Alben innerhalb der Social Networks gespeichert. Diese Alben können dann von Freunden und Bekannten betrachtet, bewertet und kommentiert, also um weitere Bedeutungsebenen angereichert werden. So kann in Communitys ein soziales Gedächtnis entstehen, Vernetzung wird Teil der Identität und bildet sie gleichzeitig ab. Allerdings, so Schmidt et al. (2009, 13), läge zumindest im Hinblick auf Fotoplattformen «nur sehr wenigen Jugendlichen» daran, Fotografien online bereitzustellen.

Neben der Pflege des eigenen Profils mit Text- und Bildinhalten kommt der Teilnahme an netzwerkinternen Gruppen Bedeutung zu. Nach Schmidt et al. (2009, 14) nutzen Jugendliche und junge Erwachsene die Funktion zur Erstellung von und Teilnahme an Gruppen, um ihre Beziehung zu anderen Personen und Themen auszudrücken, sich dadurch aber gleichzeitig auch abzugrenzen. Positionierung erfolgt also einerseits über Identifikation und andererseits über Abgrenzung. Das korrespondiert mit Interpretationen Meyens, die Gleich $(2008,153)$ darstellt: So könne Mediennutzung Menschen dazu dienen, «ihren Status innerhalb der Gesellschaft zu dokumentieren» (ebd.). Dieser Effekt könnte sich auch im Hinblick auf die Nutzung von Social Networks zeigen. Dabei geht es, wie auch bei der Vernetzung von Profilen, um aktive Beziehungspflege - dem messen Schmidt et al. $(2009,15)$ auch ausserhalb des Social Webs den Charakter einer Schlüsselqualifikation bei. Als erstes Fazit lässt sich somit festhalten, dass authentische Selbstdarstellung mittels Social Networks für jugendliche Onliner weitgehend Realität ist. Dazu nutzen sie Bildmaterial und schriftsprachliche Selbstbeschreibungen. Diese Handlungsfelder können Relevanz für eine persönliche Markenbildung haben.

\section{Identitätsbasierte Markenbildung}

Zur Analyse der Möglichkeiten von ePortfolios für eine persönliche Markenbildung im Kontext formeller Lehr- und Lernkontexte wird hier auf das Konzept der identitätsbasierten Markenbildung zurückgegriffen. Dabei wird der Begriff ‘Marke` mit Burmann/Meffert/Koers $(2005,7)$ als «Nutzenbündel mit spezifischen Merkmalen, die dafür sorgen, dass sich dieses Nutzenbündel gegenüber anderen Nutzenbündeln, welche dieselben Basisbedürfnisse erfüllen, aus Sicht der relevanten Zielgruppen nachhaltig differenziert» verstanden. Marken erfüllen somit Identifikations- und Differenzierungsfunktionen (vgl. Bergler 2007, 573f; Esch 2007, 22). Ihr Gegenstandsbereich ist nicht auf bestimmte Produktgattungen, z. B. Konsumgüter, beschränkt (vgl. Meffert/Burmann 2005, 33; Esch 2007, 19): Heute existieren 
Marken etwa in den Bereichen Dienstleistungen (Google, Allianz), Vorprodukte (INTEL, Zeiss) und Organisationen (UnICEF, AKTION MeNSCH), zudem werden auch Einzelpersonen (Heidi Klum, Stefan RaAB) zu Marken.

Die Grundidee der identitätsbasierten Markenbildung berücksichtigt als Führungskonzept die Markenidentität, das Selbstbild der internen Zielgruppe (Mitarbeiter eines Unternehmens), und auf Wirkungsebene das Markenimage, das Fremdbild bei der externen Zielgruppe (Kunden und Konsumenten), wie Burmann/Meffert (2005, 51ff; vgl. Esch/Langner/Rempel 2005) diese beschreiben. Nur aus einer klaren Identität könne auch ein klares Image entstehen, ihre Bestimmung sei eine notwendige aber keine hinreichende Massnahme für die Markenbildung, so auch Esch (2007, 81f). Dabei ist danach zu fragen, inwieweit sich die Identität eines Produkts von der eines Menschen unterscheidet. In der wirtschaftswissenschaftlichen Literatur werden verschiedene Identitätsansätze diskutiert, wobei hier der Versuch unternommen wird, die Identität eines Produkts mit menschlichen Eigenschaften zu beschreiben - letztere also auf erstere anzuwenden (vgl. Esch 2007, 95ff, Herrmann/Huber/Braunstein 2005, 184ff, Esch/Langner/Rempel 2005, 111ff). Wie etwa Herrmann/Huber/Braunstein. $(2005,188 \mathrm{ff})$ und Aaker $(2005,172)$ konstatieren, hätten Studien gezeigt, dass die Dimensionen menschlicher und produktbezogener Identität nicht vollständig übereinstimmen und eine analoge Anwendung daher mit entsprechenden Schwächen behaftet ist. Andererseits kann daher angenommen werden, identitätsbasierte Markenbildung habe einen unmittelbareren Bezug zu Individuen als zu Produkten.

Es wird davon ausgegangen, Identität entstehe bei Marken wie bei Menschen durch einen «Prozess des selbstreflexiven Denkens», Wissen und Erfahrungen aus Vergangenheit und Gegenwart würden verarbeitet (siehe Burmann/Meffert 2005, 44). Siegert (2007) beschreibt ein vergleichbares Modell wechselseitiger Bezüge. Die Konstruktion von (Marken-)Identität erfolge somit im Sinne Erik Eriksons als ständige Entwicklung, Identität sei also nicht in sich abgeschlossen, sondern dynamisch (vgl. Burmann/Meffert 2005, 43ff sowie Scherer/Wirth 2002, 339f; Abels 2006, 273ff; Abels 2007, 367ff; Hug/Hipfl 2006, 5f). Ähnliches beobachten Schmidt et al. $(2009,14)$ für die Selbstauseinandersetzung von 12- bis 24-Jährigen: Deren Selbstdarstellung (in Social Networks) sei dynamisch, «da sich die eigene Identität erst im Wechselspiel mit und Positionierung zum gesellschaftlichen Umfeld bilden kann» (ebd.). Dieser Sicht entspricht der Ansatz der identitätsbasierten Markenbildung: Als zentrale Elemente identifizieren Burmann/Meffert (2005, 43ff)

- die Wechselseitigkeit von Innen- und Aussenperspektive im Sinne von Abgrenzung einer- und Interaktion andererseits,

- die Kontinuität über einen Zeitraum, also die Beibehaltung wesentlicher Merkmale (beim Menschen z. B.: Geschlecht, Geburtstag), 
- die Konsistenz in einem Zeitpunkt, also eine widerspruchsfreie Kombination von Merkmalen, sowie schliesslich

- Individualität im Sinne einer Einmaligkeit einzelner Merkmale bzw. einzelner Kombination von Merkmalen.

Ausgehend von dieser Perspektive der Markenbildung, konstituiere sich Markenidentität aus sechs Komponenten: Herkunft, Kompetenzen, Werte, Persönlichkeit, Leistungen und Vision (Burmann/Meffert 2005, 57). Dabei lässt sich vermuten, dass diese Komponenten im wirtschaftswissenschaftlichen Kontext auch besonders im Hinblick auf die operative Ebene der Markenbildung gewählt wurden: So bilden sie ggf. nicht alle identitätsrelevanten Merkmale in vollem Umfang ab und unterliegen dadurch entsprechenden Limitationen, bieten durch eine Reduktion von Komplexität andererseits ein abgrenzbares Set an konstituierenden Komponenten. Weitere Ansätze zur Beschreibung von Markenidentität gehen von anderen Komponenten aus, wie Esch/Langner/Rempel (2005) in einer Übersicht darstellen.

Für den in diesem Beitrag gegenständlichen Einsatz von ePortfolios sollen die genannten Konstitutionselemente transferiert werden und werden daher zunächst auf Basis der Überlegungen von Burmann/Meffert (2005, 58 ff.; vgl. Burmann/Meffert/Feddersen 2007) skizziert: So sei die Herkunft einer Marke das Fundament ihrer Identität und beantworte idealiter die Frage «Woher kommen wir?». Dies sei von Bedeutung, da Marken stets im Kontext ihres regionalen, kulturellen und institutionellen Ursprungs wahrgenommen und interpretiert würden. Die Kompetenz der Marke sei ein weiterer Baustein der Markenidentität und soll die Frage «Was können wir?» beantworten. Diese bestehe - wirtschaftswissenschaftlich formuliert - in «spezifischen organisationalen Fähigkeiten [...] zur marktgerechten Kombination» von Ressourcen (Burmann/Meffert/Feddersen 2007, 6f.). Es gehe dabei um die Nutzung von Wissen und durch Regeln geleitete Handlungspotenziale mit dem Ziel, beim Nachfrager die versprochenen Leistungen zu erbringen (ebd., 7).

Die Festlegung von Werten hingegen sei eine emotionale Komponente und beantworte die Frage «Woran glauben wir?», während hingegen die Persönlichkeit einer Marke mittels menschlicher Eigenschaften beschrieben würde, ihren Ausdruck im Kommunikationsstil fände und als Antwort auf die Frage «Wie treten wir auf?» ausgerichtet sei.

Die Leistungen fänden ihre Entsprechung im symbolischen und funktionalen Kundennutzen und seien das «zentrale Verbindungselement» zwischen Identität und Soll-Image (ebd., 8). Die Beschreibung der Leistungen gäbe Antwort auf die Frage «Was tun wir?».

Der Komponente «Vision` liegt nach Burmann/Meffert $(2005,61)$ die Frage «Wohin wollen wir?» zugrunde. Inhaltliche umfasse dies die Beschreibung einer «Wunschvorstellung» (ebd.), die als erreichbar erscheinen und somit als Motivation wirken 
solle (ebd.). Hingegen seien definierte Ziele konkreter und zeitnaher erreichbar, so Burmann/Meffert (ebd.).

\section{Implikationen}

Persönliche Markenbildung setzt - ausgehend vom dargestellten Ansatz der identitätsbasierten Markenbildung - also Identität voraus. Identität schaffe Glaubwürdigkeit, weil sie klare Erwartungen generiere und diese später auch zu erfüllen vermöge, so Burmann/Meffert $(2005$, 47). Zur persönlichen Markenbildung in formellen Lehr- und Lernkontexten werden idealiter zunächst die beschriebenen Konstitutionselemente auf Identitätsebene definiert.

Herkunft («Woher komme ich?») wird dabei hier als schulische bzw. fachliche Herkunft verstanden: Verortet ein Lernender seine Fähigkeiten und Interessen - um es plakativ auf zwei Aspekte zu reduzieren - z. B. eher im naturwissenschaftlichen oder eher im künstlerischen Bereich? Im Licht des dargestellten Markenbildungsansatzes handelt es sich hier also primär um den institutionellen Ursprung.

Kompetenz («Was kann ich?») bezeichnet die Kenntnis von Regeln sowie Fähigkeiten, Aufgaben zu lösen. Im schulischen Kontext schliesst dies das Können und die Leistungsbereitschaft zum Bearbeiten von Klausuraufgaben, Vorbereiten und Halten von Referaten sowie Beantworten von Fragestellungen im Unterricht ein. Diese Kompetenzen erführen beim Übergang zum Erwachsenenalter Veränderungen, wie Schmidt et al. $(2009,16)$ darstellen: Mit Beginn von Beruf, Ausbildung oder Studium sei eine Spezialisierung zu beobachten. Dementsprechend würden spezifische Interessen vertieft und relevante Kompetenzen aufgebaut (siehe ebd.).

Unter Werte («Woran glaube ich?») können auch im Lernkontext Grundüberzeugungen des Lernenden gefasst werden. Dabei ist allerdings offen, inwieweit eine Beschränkung dieser Werte auf den Lernkontext sinnvoll und möglich ist oder, ob nicht vielmehr solche Grundüberzeugungen mittels ePortfolio abgebildet würden, die der Lernende auch ‘ausserhalb) dieses Kontextes für sich anerkennt. Die Komponente 〈Persönlichkeit〉 («Wie trete ich auf?») wird hier analog zum oben dargestellten Verständnis als Set menschlicher Eigenschaften und des Kommunikationsstils interpretiert. Vergleichbar mit der zuvor genannten Komponente (Wertes erscheint es jedoch auch hier fraglich, inwieweit eine Beschränkung dieser auf den hier gegenständlichen Kontext angemessen ist. Gleichzeitig ergäbe sich in der Anwendung die Herausforderung, Werte und Persönlichkeit in ePortfolios zu erfassen: Über diese zu sprechen und schriftlich zu definieren kann von Lernenden im Vergleich zum Erfassen von Fachinteressen oder in Form von Noten quantifizierbaren Zielen als deutlich schwieriger erlebt werden. Diese Komponenten können z. B. unter Gesichtspunkten sozialer Erwünschtheit (vgl. Brosius/Koschel 2005, $99 \mathrm{f}$.) rationalisiert und verzerrt sein. Andererseits kann eine wiederholte Auseinandersetzung damit auch Selbstreflexion und -einschätzung fördern. Möglicherweise kann dieser Prozess von Seiten des Lehrenden bzw. des ePortfolios durch bildge- 
stützte Kollagetechniken unterstützt werden, wie sie in der Markenführung bzw. -wirkungsforschung verwendet werden (vgl. Gleich 2008, 42; Kroeber-Riel/Weinberg 2003, 238f.).

Die Leistungen («Was tue ich?») beziehen sich auf die Qualität der Umsetzung von Fähigkeiten, die in der Regel über Schulnoten oder Punkte ausgedrückt werden. Was oben als «Kundennutzen» bezeichnet ist, meint hier den Grad der Vollständigkeit und Richtigkeit der Lösung von z. B. Klausuraufgaben oder der Umsetzung sonstiger Lernmassnahmen.

Der Begriff «Vision〉 («Was will ich?») schliesslich sei für den hier behandelten Bildungskontext durch den Begriff ¿Ziel〉 ersetzt. Eingeschränkt auf diesen Gegenstandsbereich könnte hier die Plangrösse «Verbessern des Notendurchschnitts», ¿Erlangen eines Abschlusses〉 oder - allgemeiner formuliert - «Positionierung als kompetenter Jugendlicher oder junger Erwachseners im Fokus liegen.

Der Ansatz der identitätsbasierten Markenbildung im skizzierten Kontext wird mit folgender Übersicht noch einmal verdeutlicht. Diese ist angelehnt an die Darstellungen von Burmann/Meffert (2005) sowie Burmann/Meffert/Feddersen (2007) und visualisiert die Zusammenhänge.

Identitätsbasierte Markenführung in formellen Lern-/Lehrkontexten

Identität

(Selbstbild)
Image

(Fremdbild)

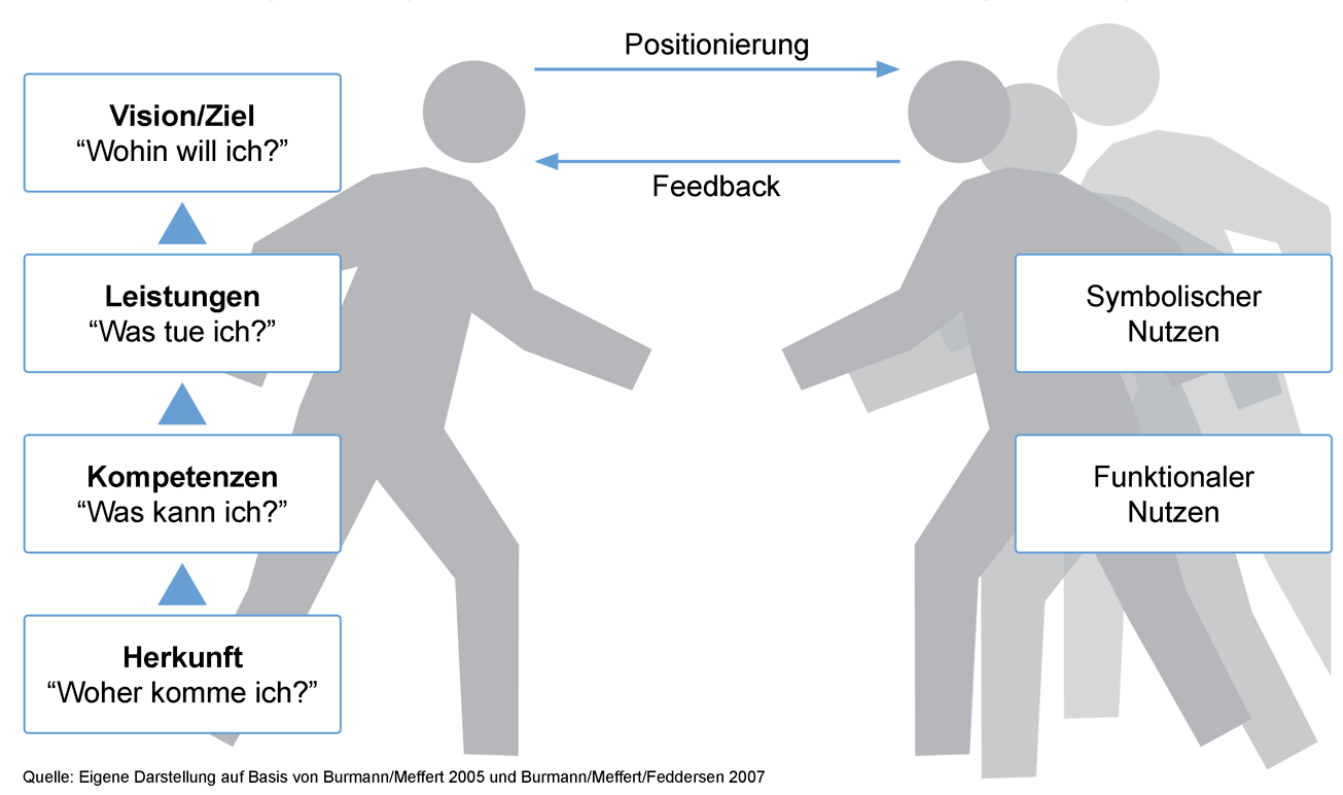

Ebenso wie persönliche Markenbildung erfordert das Engagement in Schule und Hochschule von Seiten der Lernenden, die analog zur o. g. 〈internen Zielgruppe` gesehen werden können, und Lehrenden, die als Entsprechung zur o. g. «externen 
Zielgruppes verstanden werden können, eine kontinuierliche Kontrolle, einen Abgleich der Soll- mit den Ist-Zuständen, sowie eine dementsprechenden Anpassung weiterer Handlungsschritte. Dies kann durch ePortfolios unterstützt werden: Wie dargestellt werden Social Networks und die dort verorteten Möglichkeiten der Profilierung von jugendlichen und jungen Erwachsenen genutzt. Der Umgang mit derartigen Portfolio-Anwendungen ist inzwischen also grundsätzlich bekannt und teilweise habitualisiert (vgl. van Eimeren/Frees 2008, 340 sowie Fisch/Gscheidle 2008b, $361 \mathrm{f}$.). Zur Abbildung und kontinuierlichen Beobachtung der Segmente Herkunft, Kompetenz, Werte, Persönlichkeit, Leistungen und Vision/Ziel lassen sich ePortfolios nutzen. Im Fokus ständen dabei die systematische Aufarbeitung und Darstellung der eigenen Leistungen, also Reflexion und Evaluation der eigenen Person, Lerninhalte und -erfahrungen. Dabei kann ein vierstufiges, zirkuläres Schema wie

- Zielsetzung,

- Planung,

- Realisierung und

- Kontrolle

Orientierung bieten. Auf entsprechend ausgerichteten ePortfolio-Plattformen könnten über mehrere Jahre hinweg die jeweiligen Ziele und der Grad ihres Erreichens dokumentiert werden. Entwicklungen liessen sich kontinuierlich nachvollziehen. Gleichzeitig bestünde die Möglichkeit, mit der bewussten Kombination von fachlichen Schwerpunkten und besonderen Leistungen die persönliche Markenbildung zu unterstützen. Die ständig einsehbare und kontinuierlich durchgeführte Kontrolle der eigenen Fähigkeiten und ihrer Entwicklung kann Sicherheit geben und helfen, die eigenen Leistungen auch im Vergleich zu anderen Lernenden besser einzuschätzen. Dabei kann die oben beschriebene Wechselwirkung zwischen Aussen- und Innensicht im Prozess der Markenbildung zum Tragen kommen. Social Networks unterstützen qua Konzept die o. g. Prinzipien von Portfolios gemäss Häcker (2005): Kommunikation, Transparenz und Partizipation.

Die kontinuierliche und systematisierte Auseinandersetzung mit den eigenen Leistungen kann also als Lernerfolgskontrolle dienen und Identität fördern. Das erscheint auch vor dem Hintergrund relevant, als Schmidt et al. $(2009,3)$ zu dem Ergebnis kommen, dass der Aufbau eines stabilen Selbstkonzepts für Jugendliche und junge Erwachsene «keinesfalls eine leichte Aufgabe» sei, da die Gesellschaft gegenwärtig von «einer verwirrenden Vielfalt der Lebenskonzepte und Wertesysteme» gekennzeichnet sei (ebd.).

Dieser Ansatz könnte dabei somit helfen, eigene Stärken zu erkennen, sich bewusst mit ihnen auseinanderzusetzen und diese zu kommunizieren. Andererseits können persönliche Schwächen identifiziert und systematisch aufgearbeitet wer- 
den. Das erfordert und fördert die Fähigkeit zur Selbstreflexion, die Grundlage der identitätsbasierten Markenbildung ist. Die Auseinandersetzung mit der eigenen Identität, sowie persönlichen Stärken und Schwächen mittels ePortfolios kann somit diesen Prozess unterstützen.

Ein systematisches Informations- und Berichtswesen könnte das selbstgesteuerte Lernen und dessen Überwachen unterstützen. Der aktive Kontrollprozess geht idealiter vom Lernenden aus und setzt somit voraus, dass sich dieser stets neu dafür engagiert (vgl. Häcker 2005, 5f., 7). Wie Dreer (2008) im Hinblick auf selbstgesteuertes Lernen an Berufschulen ermittelt hat, könne nicht davon ausgegangen werden, dass Lernende automatisch derartige Anforderungen erfüllen können. Würden Lehrende und auch Peers hier als Lernberater unterstützend tätig, könne das die Selbstständigkeit von Lernenden fördern, so Häcker $(2005,6)$ und Dreer $(2008$, 5). Nach Vollbrecht $(2001,65)$ könne «Eigenverantwortlichkeit für ihr [gemeint sind Lernende, Anm. des Verfassers] Lernen» durch den Einsatz interaktiver Medien im Lernkontext gestärkt werden. Der Forderung nach Interaktivität wäre durch den skizzierten Ansatz Rechnung getragen. Weiterhin, so beobachten Schmidt et al. $(2009,10)$, steige die Nutzungskompetenz von Angeboten des Social Webs mit ihrer Anwendung. Die Fähigkeit, ein eigenes Leistungsportfolio zu erstellen und zu pflegen, könnte somit durch die bereits bekannte Profilierung in Social Networks vereinfacht erfolgen. Eine Motivation zur Nutzung von ePortfolios könnte im transparenten Vergleich von Soll- und Ist-Grössen bestehen (vgl. Astleitner/Pasuchin/Wiesner 2006, 4). Dementsprechend könnten ePortfolios gleichzeitig auch mit dem Ziel eingesetzt werden, Selbstständigkeit und Medienkompetenzen zu fördern. Gemeinsam mit einer persönlichen Markenbildung in formellen Lehr- und Lernkontexten könnten sie somit mehrere Entwicklungen unterstützen.

Dabei scheint gerade die Förderung von Medienkompetenzen (zur Diskussion des Begriffs «Medienkompetenz` vgl. z. B. Hugger 2008) gegenwärtig weiter an Bedeutung zu gewinnen. So steht diese Entwicklung in Zusammenhang mit der persönlichen Markenbildung und dabei insbesondere mit der Entwicklung der eigenen Identität: Die Notwendigkeit, Informationen auszuwählen, einzuordnen und zu bewerten sowie Medien selbst zu organisieren, zu reflektieren und kreativ zu nutzen, steigt mit zunehmender 〈Informationsflut〉 (vgl. Filzmaier 2005; Moser 2006, 51 f.). So beschreibt Hugger, dass dieser Prozess in gesellschaftlichen Kontexten ablau$\mathrm{fe}$, «in denen immer weniger fest Traditionen und Autoritäten sowie klare Zielmarken der Lebensführung durchscheinen und erfahrbar werden» (Hugger 2008, 95). Diese Sicht findet sich etwa auch - wie bereits erwähnt - bei Schmidt et al. (2009, 3) sowie Scherer/Wirth $(2002,337)$.

So lässt sich zusammenfassend konstatieren, dass Jugendliche aktive Teilnehmer im Social Web sind und Social Networks routiniert, offen, intensiv und umfassend nutzen. Diese Onliner können als <early adopters bezeichnet werden, sie haben Elemente des Web 2.0 in ihren Alltag integriert und sind vom spielerischen und 
unbelasteten Umgang mit Computer und Internet geprägt (vgl. Jäckel 2005, 24; Klingler 2008, 634; Moser 2006, 56; van Eimeren/Frees 2006, 567; van Eimeren/ Frees 2008, 333). Dazu ist jedoch einschränkend anzumerken, dass diese Aussage zwar grundsätzlich Gültigkeit beanspruchen kann, Adoption und Akzeptanz von Technologien und Diensten jedoch von verschiedenen Faktoren abhängig sein können (vgl. Hugger 2008, 96; Schmidt et al. 2009, 8 ff.; Vollbrecht 2001, 63f.). Dieser Punkt ist bei der Betrachtung der Limitationen des Ansatzes zu berücksichtigen (s.u.).

Abseits dessen ist es grundsätzlich denkbar, dass Informationen aus ePortfolios mittels entsprechender Schnittstellen in andere Plattformen übertragen werden können. Als Orientierung kann Facebook Connect dienen: Ein dort registrierter Zugang kann genutzt werden, um auf andere Angebote zuzugreifen - ohne dort ein weiteres Profil anlegen zu müssen. Gleichzeitig können Aktionen, die ausserhalb des Social Networks durchgeführt werden, mittels einer plattformübergreifenden Vernetzung innerhalb des eigenen Facebook-Profils abgebildet werden. Dadurch haben Freunde und Bekannte innerhalb von Facebook die Möglichkeit, sich darüber zu informieren, wer sich gerade mit welchem Thema beschäftigt (vgl. Facebook Inc. 2009). Übertragen auf ePortfolios könnte dieses Konzept etwa für Online-Bewerbungen nutzbar gemacht werden. Zudem könnten durch die Anbindung von ePortfolios an bekannte Social Networks Adoption und Akzeptanz unterstützt werden.

Neben den genannten Chancen zeichnen sich auch Grenzen der Übertragung des identitätsbasierten Ansatzes der Markenbildung und des beschriebenen Konzepts ab. Hier sei zunächst die Betrachtung von Lernenden als «Nutzenbündel` genannt. Während dieser Terminus in der wirtschaftswissenschaftlichen Literatur in Bezug auf etwa Automobile und Konsumgüter möglicherweise regelmässig genutzt wird, erscheint er im Hinblick auf eine Übertragung auf Lehr- und Lernkontexte fragwürdig. So kann er in diesem Zusammenhang zu rational und reduzierend wirken und daher der Nutzung im ökonomischen Kontext vorbehalten bleiben.

Fraglich ist zudem auch, inwieweit sich eine strikte Unterscheidung zwischen Selbst- und Fremdbild auf den Menschen übertragen lässt. Auf Ebene der formalen Bildung wäre es denkbar, dass Lernende ihre Leistungen in einem bestimmten Kompetenzbereich selbst einschätzen (Selbstbild) und diese Sicht dann mit denen Lehrender verglichen wird (Fremdbild). Dabei ist zu berücksichtigen, dass sich diese beiden Ebenen durch den Vergleich gegenseitig beeinflussen können.

Darüber hinaus bestehen mögliche Datenschutz- und Urheberrechtsprobleme: Sind personenbezogene Informationen in ePortfolios gespeichert, können sie grundsätzlich kopiert und veröffentlicht werden - so etwa, wie es im Oktober 2009 mit einer Million Profil-Datensätze von SchülerVZ zu beobachten war. Gleichzeitig besteht die Möglichkeit, dass Nutzer fremde ePortfolios verändern. Das legt Sensibilität im Umgang mit Zugangsdaten nahe. Derartiges Wissen werde, so Schmidt 
et al. (2009, 17ff), meistens durch mediale Diskurse sowie Eltern vermittelt (mit ähnlichen Ergebnissen für 6- bis 13-Jährige: MPFS 2009) - diese Bezugsgruppen sind somit entsprechend gefordert.

Abseits dessen stellt sich die Frage, inwieweit sich ePortfolio-Vorhaben im «Pflichtprogramm Schule» (Vollbrecht 2001, 65) realisieren lassen. Während Jugendliche und junge Erwachsene Social Networks im privaten Bereich hauptsächlich «friendship-driven» nutzen, um «dabei zu sein» (Schmidt et al. 2009, 9; vgl. MPFS 2008, 55) und hier «überwiegend spassbestimmt» (Vollbrecht 2001, 65) agieren, stünde im schulischen Kontext der «ernste) Umgang im Vordergrund. Dass die Adaption privater Handlungsmuster mit pädagogischen Absichten nicht zielführend sein muss, verdeutlicht Vollbrecht $(2001,66)$ : So zeigten sich Kinder und Jugendliche "oftmals skeptisch gegenüber Versuchen, beispielsweise Fernsehinhalte in der Schule aufzuarbeiten, wenn sie sich mit ihnen stark identifizieren» (ebd.).

Liesse sich diese Kluft überwinden, könnte sich dennoch eine weitere Grenze des Ansatzes ergeben. Sind Lernende technisch wenig interessiert oder verfügen hier über geringe Kompetenzen, ist es denkbar, dass sie durch den Einsatz von ePortfolios ‘abgehängt` werden könnten. Auf der anderen Seite könnten Lernende, die unabhängig von formellen Lernkontexten technische Hilfsmittel in ihre Routinen integriert haben und diese umfassend beherrschen (z. B. «early adopters», s. o.), so zusätzlich gefördert werden. Auf diese beiden Möglichkeiten machen etwa Dreer $(2008,5)$ und Hugger $(2008,96)$ aufmerksam und plädieren dafür, das jeweilige Bildungsniveau beim Einsatz von technischen Hilfsmitteln in formellen Lernkontexten zu berücksichtigen (vgl. dazu auch z. B. Klimsa 2002).

Ungeklärt ist darüber hinaus, inwiefern in formellen Lehr- und Lernkontexten der funktionale und der emotionale Nutzen verstanden werden kann. Der Markenbildung im Bereich der Konsumgüter folgend, könnte dies bedeuten, dass Lernende durch ihre Kompetenzen einerseits Aufgabenstellungen lösen, was auf funktionaler Ebene verortet werden kann. Andererseits können sie durch wiederholte Bewältigung ähnlicher Aufgaben in der Vergangenheit Auswahlentscheidungen zu ihren Gunsten vereinfachen, da sie als «bewährt» wahrgenommen werden. Analog zum oben dargestellten Verständnis der identitätsbasierten Markenbildung wäre diese Funktion auf symbolischer Ebene zu verorten. Grundsätzlich lassen sich diese beiden Leistungsebenen folglich übertragen, jedoch erscheint der im wirtschaftswissenschaftlichen Kontext genutzte Begriff «Kundennutzen` hier unangemessen.

\section{Fazit und Ausblick}

Der Beitrag hat eine Nutzung von ePortfolios in formellen Lehr- und Lernkontexte anhand des identitätsbasierten Ansatzes der Markenbildung dargestellt. Im Hinblick auf eine systematische Auseinandersetzung mit eigenen Stärken und Schwächen, einer Förderung von Selbstreflexion und Selbstständigkeit weist dieses Konzept persönlicher Markenbildung Übereinstimmungen mit traditionellen 
Portfolioansätzen auf. Daher lässt sich konstatieren, dass ePortfolios als Instrument die persönliche Markenbildung in formellen Lehr- und Lernkontexten unterstützen können. Einordnend ist zu berücksichtigen, dass schulische Leistungen lediglich einen «Baustein` von Identität darstellen (vgl. zur Vielschichtigkeit von Markenkomponenten: Burmann/Meffert 2005, 67). Ebenso bleibt als zentrale Fragen offen, inwieweit Identität und damit Markenbildung von Produkten und Personen vergleichbar ist. Somit ist eine Betrachtung dieses eingeschränkten Kontextes gleichzeitig mit entsprechenden Limitationen im Konzept persönlicher Markenbildung verbunden. Gleichwohl kann diese Betrachtung von ePortfolios und persönlicher Markenbildung als Chance gewertet werden, Lernende auf Prozesse vorzubereiten, die im Wirtschaftskontext üblich sind: Eine strukturierte Auseinandersetzung mit der eigenen Herkunft, Kompetenzen, Werten, Persönlichkeit, Leistungen und Zielen in Form einer solch bewussten Markenbildung kann dabei helfen, die eigene Identität zu erkunden und systematisch aufzubauen.

Zudem kann die praktische Anwendbarkeit dieses Ansatzes, wie angesprochen, an Akzeptanz und Adoption durch die Lernenden scheitern. Inwieweit das der Fall ist und welche Faktoren für Nutzung und Wirkung von ePortfolios zur persönlichen Markenbildung in formellen Lehr- und Lernkontexten verantwortlich sein können, bedarf ergänzender Untersuchungen durch empirische Studien.

\section{Literatur}

Aaker, Jennifer L. «Dimensionen der Markenpersönlichkeit.» Moderne Markenführung. 4. Auflage. Hrsg. v. Franz-Rudolf Esch. Wiesbaden: Gabler, 2005. 165-176.

Abels, Heinz. Identität. Wiesbaden: VS Verlag, 2006.

Abels, Heinz. Einführung in die Soziologie. Band 2: Die Individuen in ihrer Gesellschaft. 3. Auflage. Wiesbaden: VS Verlag, 2007.

Astleitner, Hermann; Pasuchin, Iwan; Wiesner, Christian. «Multimedia und Motivation - Modelle der Motivationspsychologie als Grundlage für die didaktische Mediengestaltung.» MedienPädagogik (22.03.2006): www.medienpaed.com/2006/astleitner0603.pdf.

Bergler, Reinhold. «Psychologie der Markenführung: Von den Anfängen zur gegenwärtigen Problemlage.»Psychologie der Markenführung. Hrsg. v Arnd Florack, Martin Scarabis und Ernst Primosch. München: Franz Vahlen, 2007. 573-620.

Burmann, Christoph; Meffert, Heribert. «Theoretisches Grundkonzept der identitätsorientierten Markenführung.» Markenmanagement. 2. Auflage. Hrsg. v. Heribert Meffert, Christoph Burmann und Martin Koers. Wiesbaden: Gabler, 2005. 37-72.

Burmann, Christoph; Meffert, Heribert; Feddersen, Christian. «Identitätsbasierte Markenführung.»Psychologie der Markenführung. 2. Auflage. Hrsg. v. Arnd Florack, Martin Scarabis und Ernst Primosch. München: Franz Vahlen, 2007. 3-30.

Burmann, Christoph; Meffert, Heribert; Koers, Martin. «Stellenwert und Gegenstand des Markenmanagements.» Markenmanagement. 2. Auflage. Hrsg. v. Heribert Meffert, Christoph Burmann und Martin Koers. Wiesbaden: Gabler, 2005. 3-17.

Brosius, Hans-Bernd; Koschel, Friederike. Methoden der empirischen Kommunikationsforschung. 3. Auflage. Wiesbaden: VS Verlag, 2005. 
Dreer, Silvia. «E-Learning als Möglichkeit zur Unterstützung des selbstgesteuerten Lernens an Berufsschulen.» MedienPädagogik (12.06.2008): www.medienpaed.com/2008/dreer0806.pdf.

Eble, Michael J. «Phänomene des Web 2.0: Fehlt deutschen Zeitungen eine jugendliche Perspektive?»Phänomene des Web 2.0 als Agenten des kulturellen Wandels. Sonderheft SPIEL (= Siegener Periodicum zur Internationalen Empirischen Literaturwissenschaft). Hrsg. v. Daniela Pscheida und Sascha Trültzsch. Frankfurt a.M. u. a.: Peter Lang, 2009. [im Erscheinen]

Eimeren, Birgit van; Frees, Beate. «Zukünftige Medien: Praxistauglich für den Konsumenten.» Media Perspektiven 11 (2006): 563-571.

Eimeren, Birgit van; Frees, Beate. «Internetverbreitung: Grösster Zuwachs bei Silver-Surfern.» Media Perspektiven 7 (2008): 330-344.

Esch, Franz-Rudolf. Strategie und Technik der Markenführung. 4. Auflage. München: Franz Vahlen, 2007.

Esch, Franz-Rudolf; Langner, Tobias; Rempel, Jan Eric. «Ansätze zur Erfassung und Entwicklung der Markenidentität. » Moderne Markenführung. 4. Auflage. Hrsg. v. Esch, FranzRudolf Esch. Wiesbaden: Gabler, 2005. 103-129.

Feierabend, Sabine;Kutteroff, Albrecht. «Medien im Alltag. Jugendlicher - multimedial und multifunktional.» Media Perspektiven 12 (2008): 612-624.

Filzmaier, Peter. «(K)eine neue Medienkompetenz für das Internet?» Bundeszentrale für politische Bildung (2005): www.bpb.de/themen/ZUOHC7.html.

Fisch, Martin; Gscheidle, Christoph. «Technische Ausstattung der Onliner in Deutschland.» Media Perspektiven 7 (2008a): 345-349.

Fisch, Martin; Gscheidle, Christoph. «Mitmachnetz Web 2.0: Rege Beteiligung nur in Communitys.» Media Perspektiven 7 (2008b): 356-364.

Gleich, Uli. «Konsumentenforschung.» Media Perspektiven 1 (2008): 40-46.

Haas, Sabine; Trump, Thilo; Gerhards, Maria; Klingler, Walter. «Web 2.0: Nutzung und Nutzertypen.» Media Perspektiven 4 (2007): 215-222.

Häcker, Thomas. «Portfolio als Instrument der Kompetenzdarstellung und reflexiven Lernprozesssteuerung.» Berufs- und Wirtschaftspädagogik - online (Nr. 8, 2005): www.bwpat. de/ausgabe8/haecker_bwpat8.pdf.

Herrmann, Andreas; Huber, Frank; Braunstein, Christine. "Gestaltung der Markenpersönlichkeit mittels der «means-end»-Theorie.» Moderne Markenführung. 4. Auflage. Hrsg. v. Franz-Rudolf Esch. Wiesbaden: Gabler, 2005. 177-207.

Höflich, Joachim R.; Gebhardt, Julian. «Der Computer als Kontakt- und Beziehungsmedium. Theoretische Verortung und explorative Erkundungen am Beispiel des Online-Chats.» Medien \& Kommunikationswissenschaft 1 (2001): 24-43.

Hug, Theo; Hipfl, Brigitte. «Medien-Gemeinschaften: Aktuelle Diskurse und konzeptionelle Analysen.» MedienPädagogik (26.04.2006): www.medienpaed.com/2006/hug_hipfl0604. pdf.

Hugger, Kai-Uwe. «Medienkompetenz.» Handbuch Medienpädagogik. Hrsg. v. Uwe Sander, Friederike von Gross und Kai-Uwe Hugger. Wiesbaden: VS Verlag, 2008. 93-99.

Jäckel, Michael. Medienwirkungen. 3. Auflage. Wiesbaden: VS Verlag, 2005.

Klimsa, Paul. «Multimedianutzung aus psychologischer und didaktischer Sicht.» Information und Lernen mit Multimedia und dem Internet: Lehrbuch für Studium und Praxis. 3. Auflage. Hrsg. v. Ludwig J. Issing und Paul Klimsa. Weinheim: BeltzPVU, 2002. 5-17.

Klingler, Walter. «Jugendliche und ihre Mediennutzung 1998 bis 2008.» Media Perspektiven 12 (2008): 625-634. 
Kroeber-Riel, Werner; Weinberg, Peter. Konsumentenverhalten. 8. Auflage. München: Franz Vahlen, 2003.

Medienpädagogischer Forschungsverbund Südwest (MPFS). JIM '98: Jugend, Information, (Multi-)Media. Baden-Baden: Medienpädagogischer Forschungsverbund Südwest, 1998.

Medienpädagogischer Forschungsverbund Südwest (MPFS). JIM-Studie 2008: Jugend, Information, (Multi-)Media. Stuttgart: Medienpädagogischer Forschungsverbund Südwest, 2008.

Medienpädagogischer Forschungsverbund Südwest (MPFS). KIM-Studie 2008: Kinder + Medien, Computer + Internet. Stuttgart: Medienpädagogischer Forschungsverbund Südwest, 2009.

Meffert, Heribert; Burmann, Christoph. «Wandel in der Markenführung - vom instrumentellen zum identitätsorientierten Markenverständnis.» Markenmanagement. 2. Auflage. Hrsg. v. Heribert Meffert, Christoph Burmann und Martin Koers. Wiesbaden: Gabler, 2005. 19-36.

Moser, Heinz. Einführung in die Medienpädagogik. 4. Auflage. Wiesbaden: VS Verlag, 2006.

Scherer, Helmut; Wirth, Werner. «Ich chatte - wer bin ich? Identität und Selbstdarstellung in virtuellen Kommunikationssituationen.» Medien \& Kommunikationswissenschaft 3 (2002): 337-358.

Schmidt, Jan-Hinrik; Paus-Hasebrink, Ingrid; Hasebrink, Uwe; Lampert, Claudia. «Heranwachsen mit dem Social Web.» Hamburg/Salzburg. http://www.lfm-nrw.de/downloads/ zusammenfassung_socialweb.pdf.

Siegert, Gabriele. «Werbung und Konsum: Marken als zweiseitiger, zweidimensionaler Kommunikationsprozess.» Ambivalenzen des Konsums und der werblichen Kommunikation. Hrsg. von Michael Jäckel. Wiesbaden: VS Verlag, 2007. 109-125.

Stegbauer, Christian. "Soziale Netzwerkanalyse.» Handbuch Medienpädagogik. Hrsg. v. Uwe Sander, Friederike von Gross und Kai-Uwe Hugger. Wiesbaden: VS Verlag, 2008. 166-172.

Vollbrecht, Ralf. Einführung in die Medienpädagogik. Weinheim und Basel: Beltz, 2001.

Wirtz, Bernd W. «Deutschland Online 5.» Deutschland Online (2008): www.studie-deutschland-online.de.

\section{Quellen}

Bundesverband Informationswirtschaft, Telekommunikation und neue Medien (BITKOM). "Kinder und Jugendliche im Internet.»BITKOM (2009): www.bitkom.org/files/documents/BITKOM_Praesentation_Jugend_im_Web_07_07_2009_PRESSE.pdf.

Facebook Inc. «Facebook Connect» Facebook Inc. (2009): developers.facebook.com/connect.php.

Xing AG. «Corporate Pages I Xing AG.» Xing AG (2009): corporate.xing.com. 